\title{
Circular Dichroism of Bipolarons in a Chiroptically Active Conjugated Polymer
}

\author{
Hiromasa Goto \\ Division of Materials Science, Faculty of Pure and Applied Sciences, University of \\ Tsukuba, Tsukuba, Ibaraki 305-8573, Japan \\ Correspondence to H. Goto, Email: gotoh@ims.tsukuba.ac.jp
}

Keywords bipolarons, chiroptical activity, electromagnetic chiroptical effect, liquid crystal

\begin{abstract}
Bipolarons (dications), as charge carriers in chiroptically active poly(3,4-ethylenedioxythiophene) (PEDOT*, * = chiral, atropisomer) prepared in cholesteric liquid crystals, were examined by circular dichroism (CD) spectroscopy. PEDOT* showed chiroptical activity in the near-IR (NIR) range indicating the existence of bipolarons with helical structure. The main-chain helicity and fingerprint texture derived from helical aggregation of the polymer at the macroscopic level are produced by the transcription from liquid crystals during the polymerization process. This is referred to as a matrix imprinting (MAT-I) process. The chiral-bipolaron (chiral-dications) state was confirmed through NIR-CD. The chiroptical activity of the bipolarons comes from the main-chain helical structure at the molecular level. A modulation of main-chain helicity due to generation of the polarons and bipolarons results in the electrochemically driven change in chiroptical activity (electro-chiroptical effect). We refer to chiroptically active charge carriers in the PEDOT* as chiralions.
\end{abstract}

\section{Introduction}

Electroactive $\pi$-conjugated polymers, such as polythiophene, and their derivatives, show electrochromism. ${ }^{[1-3]}$ The change in color of the electroactive $\pi$-conjugated polymers are due to the generation of polarons (radical cations) and bipolarons (dications). Doping leads to changes in the effective conjugation length and the charge species of the polymers, resulting in modifications to the chromophores along the main-chain. Electrochemical processes can precisely tune the electronic state of the polymer through electrochemical redox by applying a low voltage. A change in the electronic state of the chirally conjugated polymers also can tune the optical activities. The asymmetric electrochemical polymerization in chiral liquid crystals, developed by the author, produces electro-chiroptically active polymers from achiral monomers as a new type of asymmetric synthesis. ${ }^{[4]}$

Central chirality of molecules with liquid crystallinity can form cholesteric (or chiral nematic, $\mathrm{N}^{*}$ ) liquid crystals with helical aggregation. The electrochemical polymerization in the cholesteric liquid crystal electrolyte solution progresses with molecular structure transcription of the helical structure of the cholesteric liquid crystal that results in polymers possessing helical structures. The macroscopic structure 


\section{Bipolaron Circular Dichroism in PEDOT*}

(fingerprint texture) of the resultant polymer comes from matrix imprinting of the fingerprint texture of the cholesteric liquid crystal. The transcription by matrix imprinting is carried out from the molecular level to the macroscopic level with hierarchical molecular transcription.

The polymers thus prepared in liquid crystals show an electrochemically driven dynamic change in chiroptical activity. We call this effect "electro-chiroptical chromism".

Furthermore, electrochemical polymerization in crystals was carried out in order to obtain crystalline polymers. ${ }^{[5]}$ Organizing environments, such as crystals and liquid crystals, function as reaction matrices possessing a certain order. ${ }^{[6]}$ These polymerizations will be referred to as structure-organizing polymerizations (SOP) by matrix imprinting (MAT-I). SOP produces a 3D organized structure by a bottom-up technique.

To date, optical activities of bipolarons in chiral conjugated polymers have not been examined, although we have described the optical activity of polarons in a helical architecture of chiral conjugated polymers. ${ }^{[7]}$ In this report, the circular dichroism (CD) of doped (electrochemical doping of the electron acceptor, perchlorate ion) and dedoped (electrochemical dedoping of the acceptor) chiroptically active poly $(3,4$-ethylenedioxythiophene) (PEDOT $*, *=$ chirality), prepared in a cholesteric liquid crystal, in a near-IR (NIR) range was examined. Electrochemical doping of the perchlorate ions removes electrons from the PEDOT* to form a doped state. Therefore, doping is equivalent to oxidation.

\section{Experimental}

Materials

4- $n$-Hexyl-4'-cyanobiphenyl (abbreviated as 6CB, nematic liquid crystal) (Merck, KGA, Darmstadt, Germany), cholesteryl pelargonate (chiral inducer or chiral dopant) (Tokyo Chemical Industry, Japan), tetrabutyl ammonium perchlorate (TBAP, supporting electrolyte) (Tokyo Chemical Industry, Japan) were used for preparation of cholesteric liquid crystal electrolyte solutions.

Preparation of poly(3,4-ethylenedioxythiphene) (PEDOT)

2,3,2',3',2",3"-Hexahydro[5,5':7',5"]ter(thieno[3,4-b][1,4]dioxine) [terEDOT] has been prepared via several routes. ${ }^{[8-10]}$ In this study, terEDOT as a monomer was synthesized with the Grignard coupling reaction between 3,4-ethylenedioxythiophene (2,3-dihydrothieno[3,4-b]-1,4-dioxin, abbreviated as EDOT) and 5,7-dibromo-2,3-dihydro-thieno[3,4-b][1,4]dioxine (dibromo-EDOT), according to the previously reported method. ${ }^{[11]}$ First, bromination of 5,7-dibromo-2,3-dihydro-thieno[3,4-b][1,4]dioxine with N-bromosuccinimide (NBS) yielded dibromo-EDOT. ${ }^{[12]}$ Then, the Grignard coupling reaction of EDOT and dibromo-EDOT, with the aid of a Ni(II) catalyst, produced terEDOT as a monomer. A thin film of poly(terEDOT) (abbreviated here as PEDOT*) was prepared by electrochemical polymerization of the terEDOT in a cholesteric liquid crystal solution (Scheme 1), as described below. ${ }^{[11]}$

The cholesteric liquid crystal electrolyte solution was prepared by addition of TBAP (supporting salt, $1 \mathrm{mg}$ ), terEDOT (monomer, $20 \mathrm{mg}$ ), and cholesteryl pelargonate (chiral inducer, $20 \mathrm{mg}$ ) to $6 \mathrm{CB}$ as a solvent. The total weight of the liquid crystal 


\section{Bipolaron Circular Dichroism in PEDOT*}

solution was adjusted to be $0.5 \mathrm{~g}$. The electrochemical polymerization was carried out by application of $3 \mathrm{~V}$ across a sandwich-type polymerization cell consisting of two indium-tin-oxide (ITO) coated glass electrodes and a Teflon sheet as a spacer charged with the cholesteric liquid crystal electrolyte solution containing monomer (cell gap = $0.2 \mathrm{~mm}$ ). The PEDOT* was obtained in the form of a film deposited on the ITO glass at the anode side. In this experiment, polymerization was carried out for $5 \mathrm{~min}$ at $20^{\circ} \mathrm{C}$. Polymerization at this lower temperature produced short helical pitches in the cholesteric liquid crystal electrolyte solution, resulting in the formation of the polymer with intense optical activity and higher crystallinity compared to the sample from our previous attempt (polymerization at $25^{\circ} \mathrm{C}$ ). ${ }^{[11]}$ As prepared, PEDOT* is the doped state, with perchlorate ions, because the electrochemical polymerization progressed with electrochemical doping. The dedoped state of the PEDOT* (PEDOT) was electrochemically prepared in $0.1 \mathrm{M}$ TBAP/acetonitrile solution with application of -0.8 $\mathrm{V}$ vs. the $\mathrm{Ag} / \mathrm{Ag}^{+}$reference electrode.

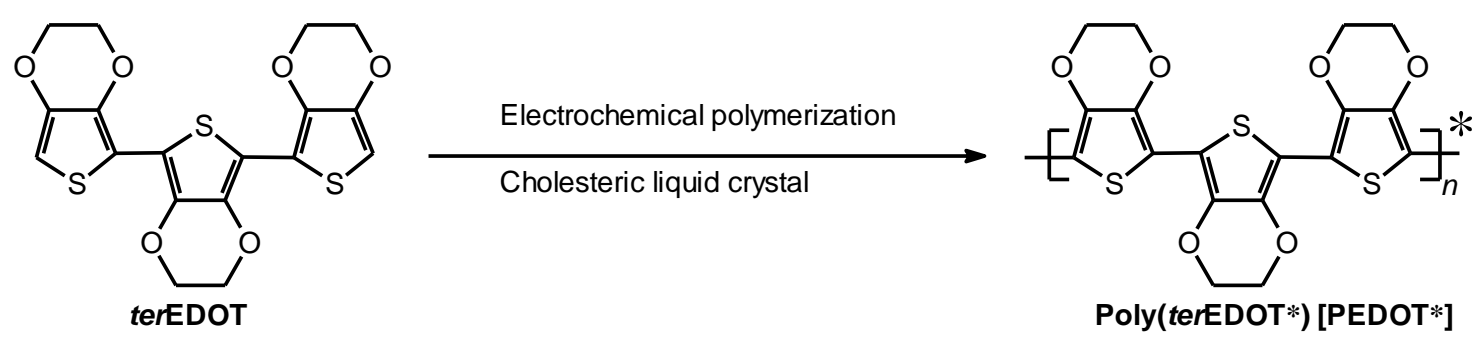

Scheme 1. Preparation of PEDOT*, poly[2,3,2',3',2",3"-hexahydro[5,5':7',5"]ter(thieno[3,4-b][1,4]-dioxine)] (poly $\left(\right.$ terEDOT*), in cholesteric liquid crystal. ${ }^{[11]}$

\section{Characterization}

The CD spectra of the PEDOT* at short wavelengths were obtained with a Jasco J-720 spectrometer (Japan). The CD spectra of the PEDOT* at long wavelengths were obtained over two ranges, 800-1500 nm and 1500-2000 nm, using a custom Jasco J-730 (Japan). The data in these ranges were combined to show the entire CD spectra. Linear dichroism was checked by the JASCO J-730 and an artifact detection/offset system (JASCO). The scanning electron microscopy (SEM) observations were carried out using a JSM-5610 microscope (JEOL Co. Ltd., Japan). Because the PEDOT* thin films possessed conductivity, observation of the PEDOT* could be performed without Au or carbon coating. The circularly polarized differential interference contrast microscopy (C-DIM) observation was performed using an ECLIPSE LV100 optical microscope with a circularly polarized optical unit (Nikon, Japan).

\section{Results and Discussion}

The electrochemical polymerization in the cholesteric liquid crystal yielded a

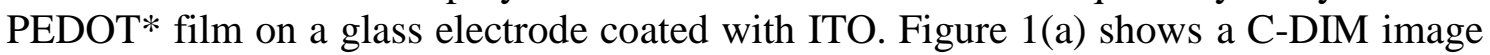
of the film surface. The SEM observations revealed that the "fingerprint texture" consisted of a 3-D structure apparently produced by the matrix imprinting, Figs. 1(b) and (c). 

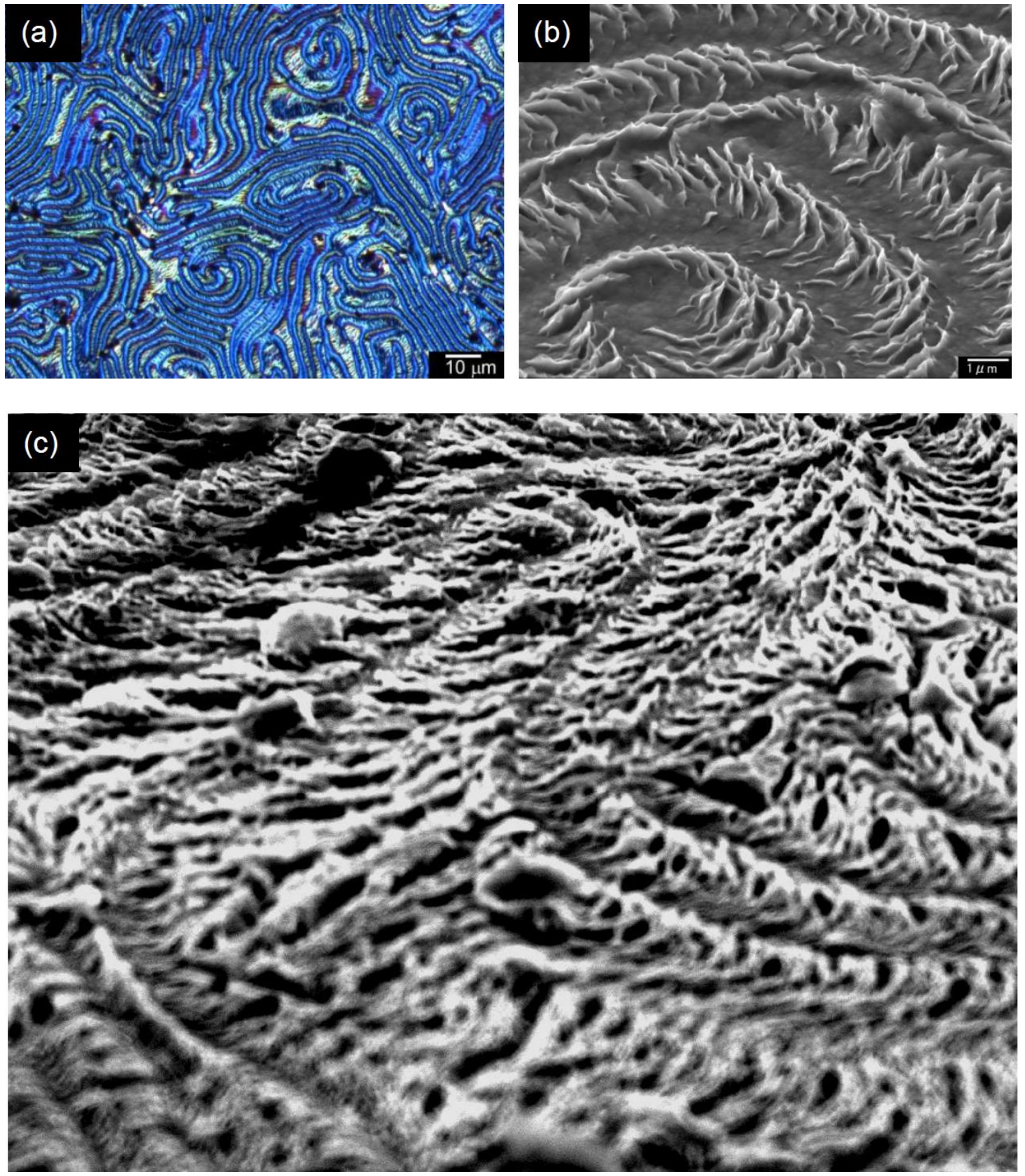

Figure 1. Surface image of the chiral poly(3,4-ethylenedioxythuiophene) (PEDOT*) prepared in a cholesteric liquid crystal electrolyte solution. (a) Circularly polarized differential interference contrast microscopy image (C-DIM); (b) Scanning electron microscopy (SEM) image; (c) SEM image from an oblique direction.

Figure 2 shows plausible chemical structures of the neutral form, Fig. 2(a), polarons, Fig. 2(b), and bipolarons, Fig. 2(c), of an achiral PEDOT prepared in a common organic solvent, as examples. The structural change to partly quinonoid sequence from benzenoid sequence in the main-chain is reproducible by the redox process.

Figure 3 displays the CD spectra of the doped and dedoped PEDOT*. The ellipticity was changed upon doping. The dedoped sample in this study can be 


\section{Bipolaron Circular Dichroism in PEDOT*}

considered to be in an intermediate doped state (lightly doped) because the sample had residual perchlorate ions. The dedoped (lightly doped) sample showed a positive signal at short wavelength $(517 \mathrm{~nm}, 2.4 \mathrm{eV})$ due to its main-chain helical structure, although the CD band due to the helical main-chain was observed at $465 \mathrm{~nm}$ for another sample obtained in our previous research. This may be due to the fact that polymerization in a cholesteric liquid crystal at lower temperature improved crystallinity of the resultant PEDOT*. Enhanced molecular packing of the PEDOT* may allow low-energy optical transitions of the main-chain.

a)

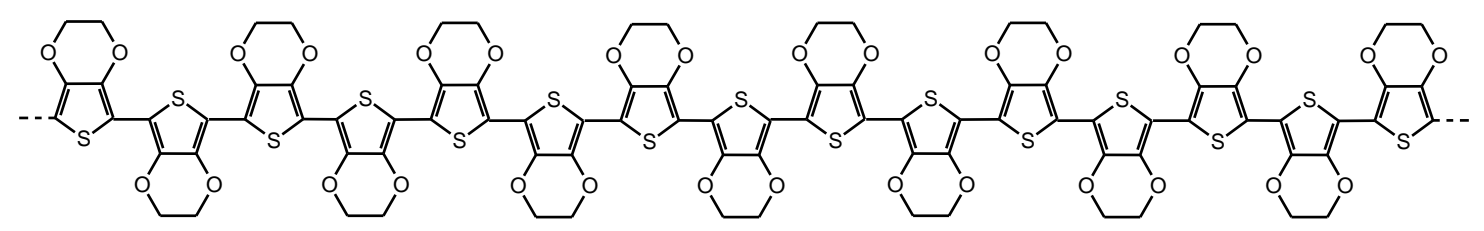

b)

$$
+e^{-} \mid-e^{-}
$$

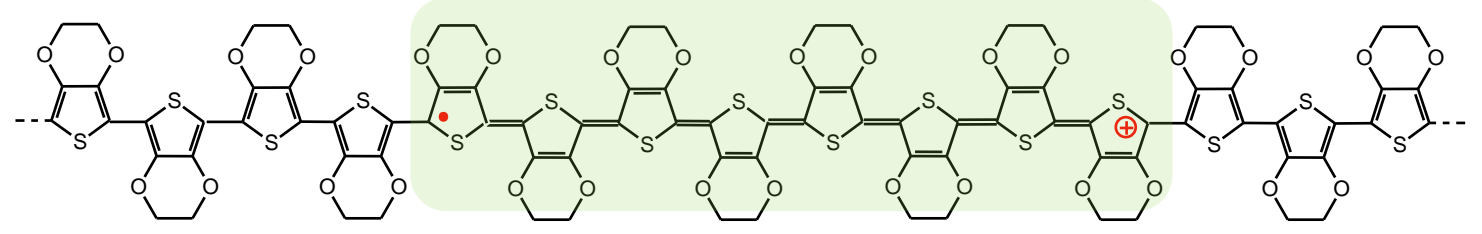

Polarons

$$
+e^{-}||-e^{-}
$$

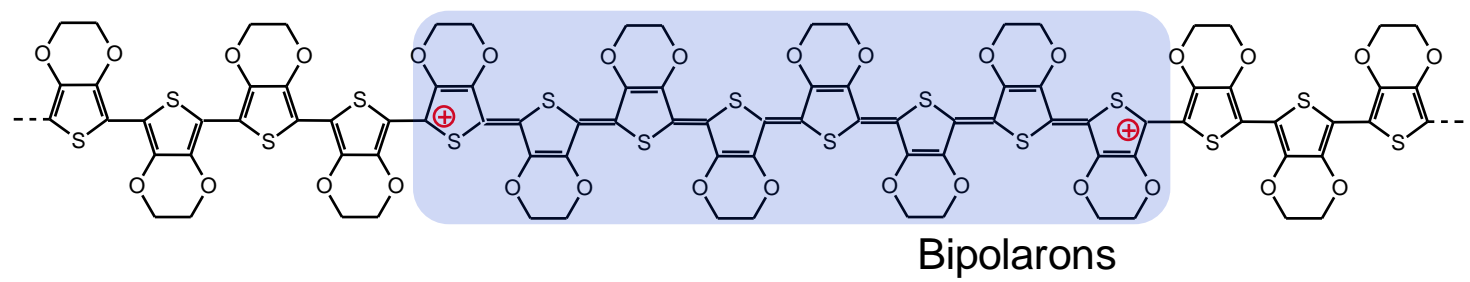

Figure 2. (a) Chemical structure of poly(3,4-ethylenedioxythiophene) (PEDOT) prepared in an isotropic solution (general method) (not liquid crystal solution); (b) polarons (radical cations); (c) bipolarons (dications).

The positive signal in the CD at $900-1000 \mathrm{~nm}(1.24-1.38 \mathrm{eV})$ is suggested to be due to a polaron band of the dedoped (slightly doped) PEDOT*, Fig. 3, and Fig. 3, inset (b). The positive signal in the CD in the NIR range $(>1000 \mathrm{~nm},>1.24 \mathrm{eV}$, low energy range) is due to generation of bipolarons in the PEDOT*, Fig 3, and Fig. 3 inset (b). On the other hand, the dedoped (slightly doped) PEDOT* shows a broad negative CD signal in the NIR range at low energy, indicating that the dedoped sample also has bipolarons in the main-chain. The sign of the CD signal of the PEDOT* in the NIR range was inverted upon electrochemical doping. In our previous research, inversion of the sign of the Cotton effect upon doping was observed in the IR-NIR range $(<1000 \mathrm{~nm}$, polaron band). ${ }^{[11]}$ The present research indicates that chirality of bipolarons can be tuned 


\section{Bipolaron Circular Dichroism in PEDOT*}

with doping level. In this case, the helicity of the main-chain and the chiral aggregation form were changed upon doping-dedoping. In this measurement, the detection gain (sensitivity) of the instrument was turned down (x1/4) because the sample showed intense signals in the CD. The CD signals (ellipticity) in Fig. 3 are normalized values.

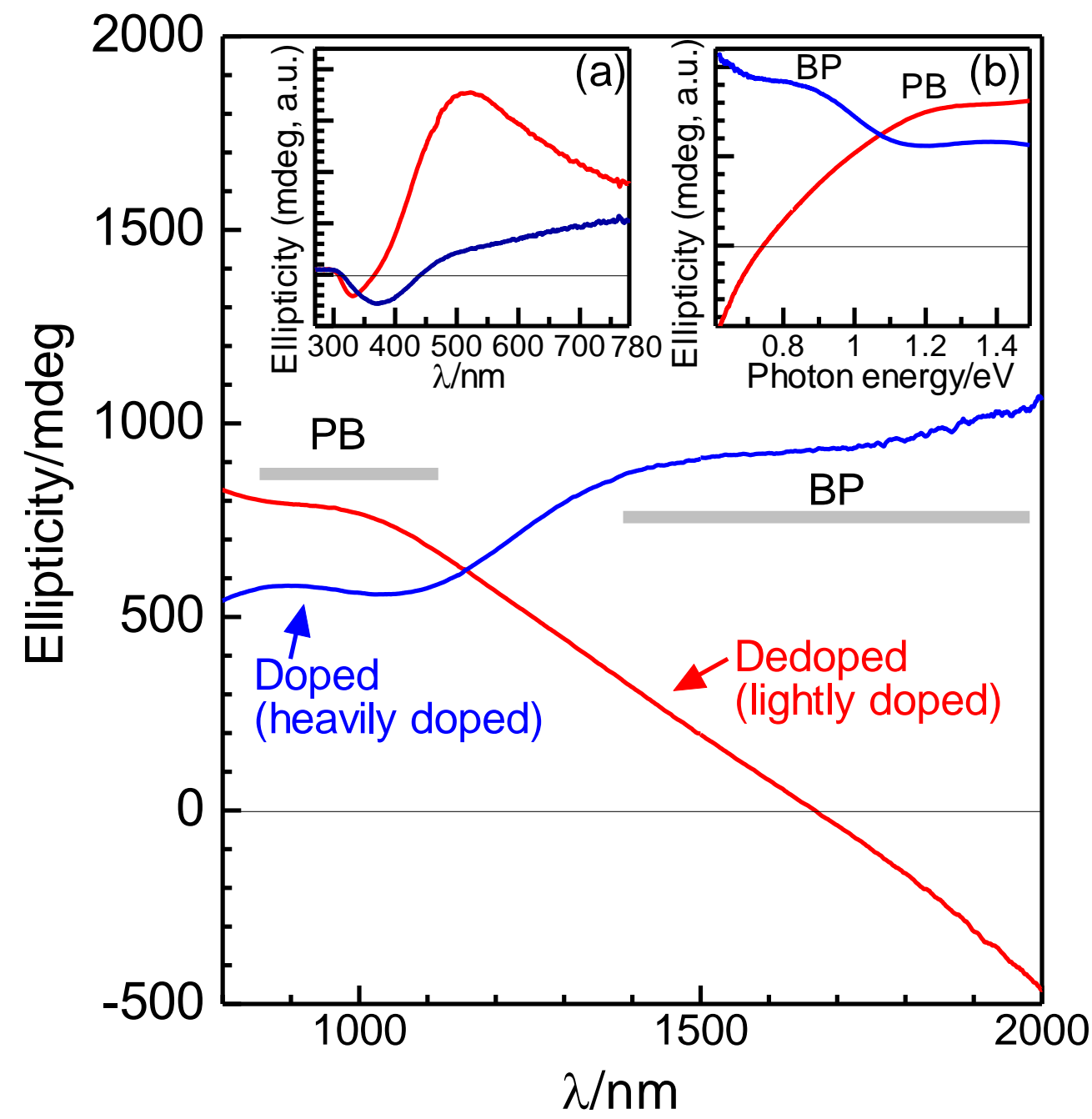

Figure 3. Circular dichroism absorption (CD) spectra of the PEDOT* of the doped and the dedoped forms. Inset (a): ellipticity vs. $\lambda$ at short wavelengths; inset (b): ellipticity vs. photon energy, $\mathrm{PB}=$ polarons band, $\mathrm{BP}=$ bipolarons band.

Models

Figure 4 shows a model of the electrochemical dedoping, Fig. 4(a), and doping, Fig. 4(b), for the PEDOT*. The direction of the current drives injection and removal of the dopant ions. The electrochemical doping process tunes the color of the PEDOT* through generation of polarons and bipolarons as charge carriers. However, the surface structure, Fig. 4(c), was apparently unaffected by the redox (doping-dedoping) process.

Because the helical structure of the main-chain can not be directly observed at the molecular level, possible aggregation forms and the main-chain structures of the PEDOT* $^{*}$ prepared in the cholesteric liquid crystal are proposed. Neighboring monomer repeat units in the main-chain of a PEDOT prepared by the general method in an isotropic solvent can be pointed in opposite direction because of intramolecular 


\section{Bipolaron Circular Dichroism in PEDOT*}

non-covalent interaction between the oxygen and sulfur atoms in the neighboring thiophene units, ${ }^{[13,14]}$ and steric hindrance of unshared electron pairs on the oxygen (oxygen-oxygen interaction) in the neighboring thiophene units. The role of the nonbonding interaction in aromatic type conjugated polymers has been theoretically studied. ${ }^{[15]}$ In the case of PEDOT, the steric interaction mainly affects the conformation.

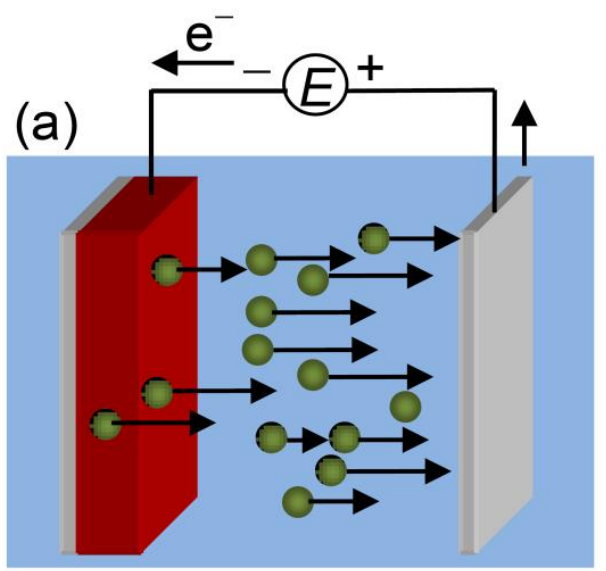

\section{Dedoping}

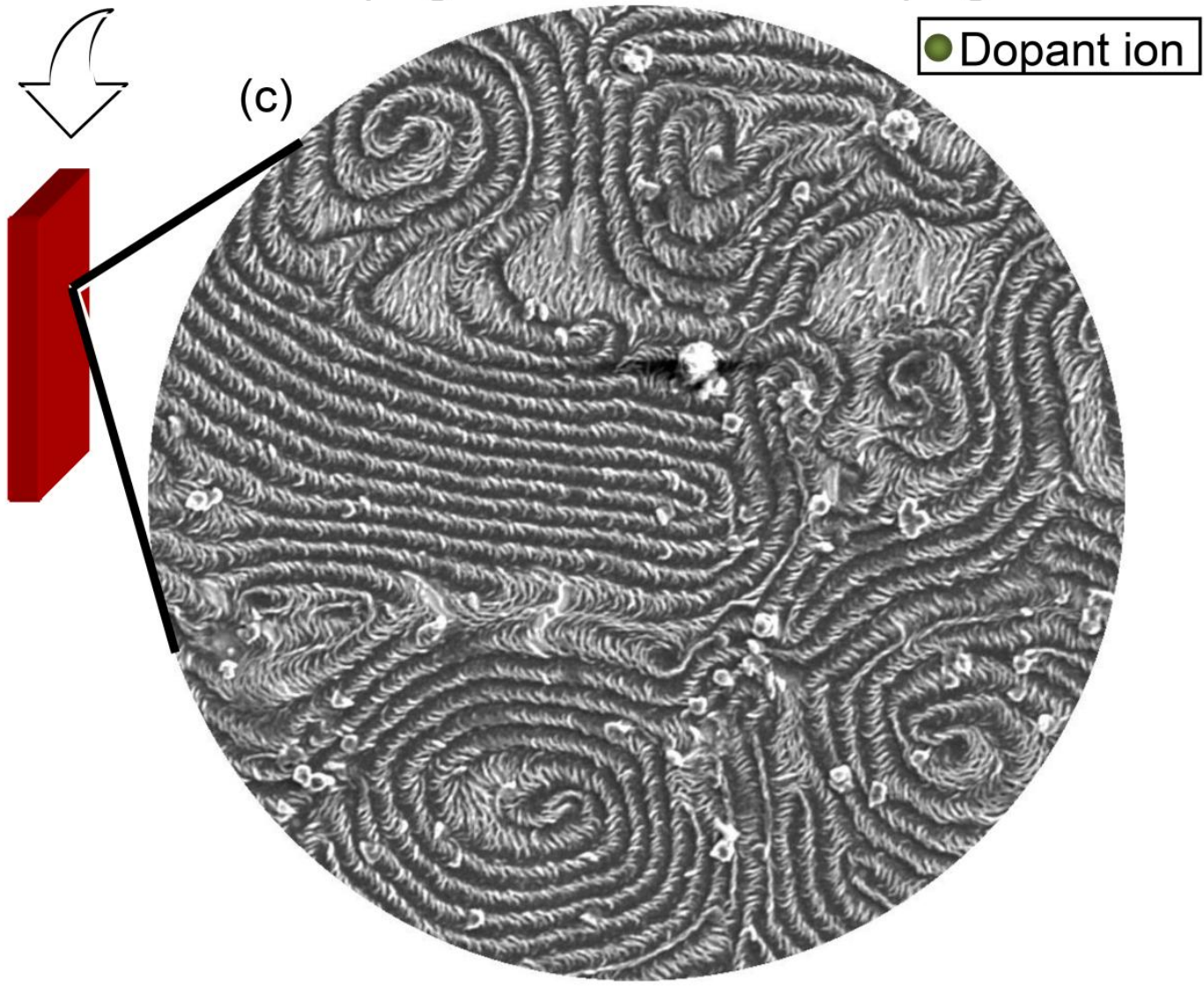

Figure 4. (a): Electrochemical dedoping. (b): Doping. (c): SEM image of the PEDOT* film prepared in cholesteric liquid crystals on (ITO)-coated glass electrode (as prepared sample, doped form). Dopant ion $=\mathrm{ClO}_{4}{ }^{-}$. 


\section{Bipolaron Circular Dichroism in PEDOT*}

The PEDOT* ${ }^{*}$ synthesized in a cholesteric liquid crystal medium are suggested to form predominantly one-handed main-chain helical structures, helically formed inter-main-chain aggregates, and coil. Here, three possible forms of sequences of the 3,4-ethylenedioxyhiophene (EDOT) unit in the main-chain are considered: EDOT units point in opposite directions and gently twist to form a helical structure, Fig. 5(I); EDOT units gently twist in one-handed screw direction, Fig. 5(II); three 3,4-ethylene dioxyhiophene (EDOT) units as monomer repeat units (terEDOT) gently twist in one direction to form a helical structure, Fig. 5(III). The monomer unit consisting of the triple-EDOT functions as one group. In these cases (I-III), the monomer can be pre-oriented in cholesteric liquid crystal along the helical structure of the cholesterics before polymerization. Transcription of the liquid crystal form is carried out in the polymerization.

I)

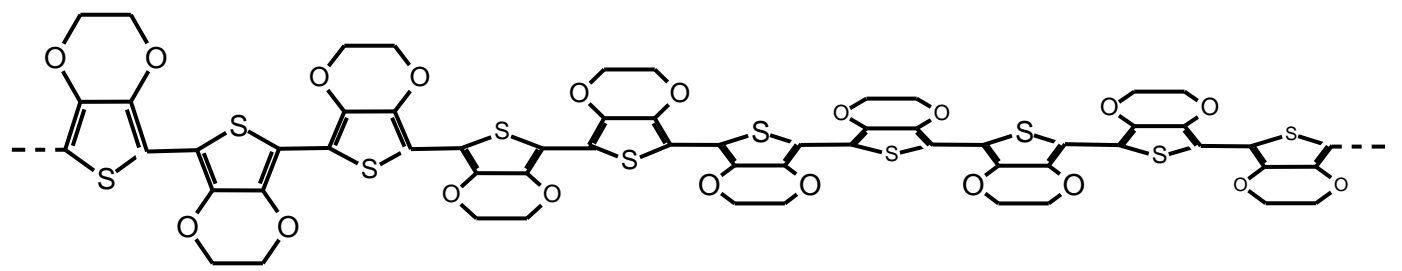

II)

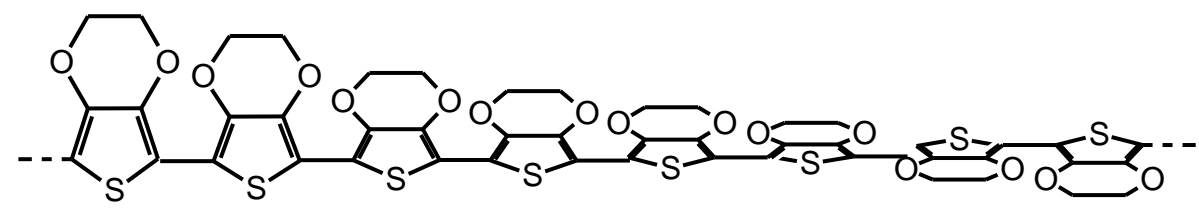

III)

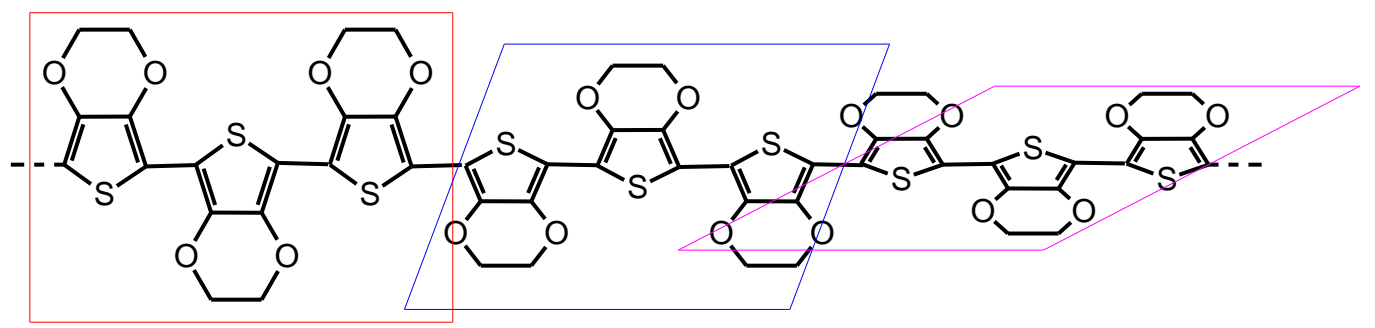

Figure 5. Possible structure of chiroptically active poly(3,4-dioxythiophene)(PEDOT*) prepared in cholesteric liquid crystal. I) neighboring 3,4-dioxythiophene (EDOT) units point in opposite directions to form helical structure; II) EDOT units point in the same direction and gently twist in one-handed direction; III) Three EDOT units as a monomer repeat unit (terEDOT) gently twist in one-direction to form helical structure.

Four structures of bipolarons in the PEDOT* upon doping are proposed. As shown in Fig. 6(A), the neighboring EDOT units point in opposite directions in a given molecule to form a planar structure. In this case, the quinonoid sequence shows no optical activity. Two charges exist in the sequence. In addition, as shown in Fig. 6(B), a form in which EDOT units gently twist in a one-handed direction can be considered. Lastly, a bipolaron form in which the neighboring EDOT units or three-EDOT units (monomer repeat unit) point in opposite directions and gently twist to form helical structures is possible, Figs. 6(C,D). 


\section{Bipolaron Circular Dichroism in PEDOT*}

The sequence of planar quinonoid structures can twist quite gently in a one-handed direction to form a helical structure of the main-chain as an atropisomer (Fig. 6(B-D)). The small dihedral angle between neighboring EDOT units can be changed through doping. Quinoid coil continuum can be also plausible. ${ }^{[16]}$ In this case, the coil pitch is tuned by the doping-dedoping.

A)

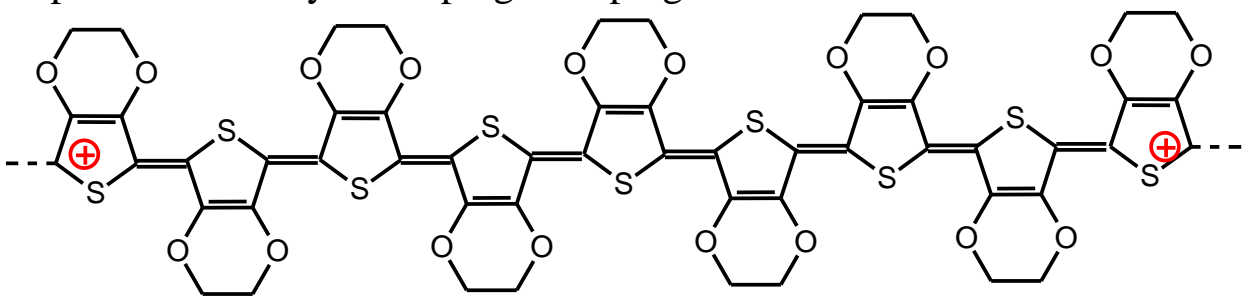

B)

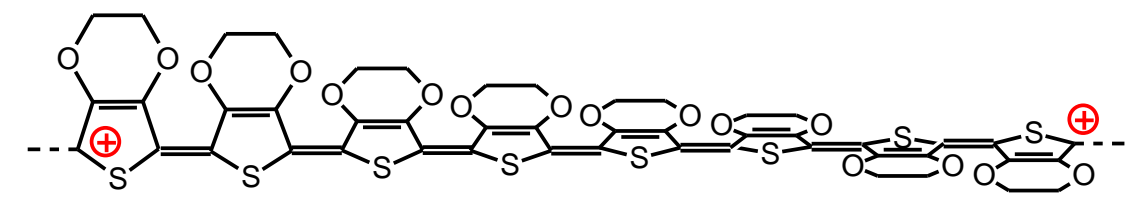

C)

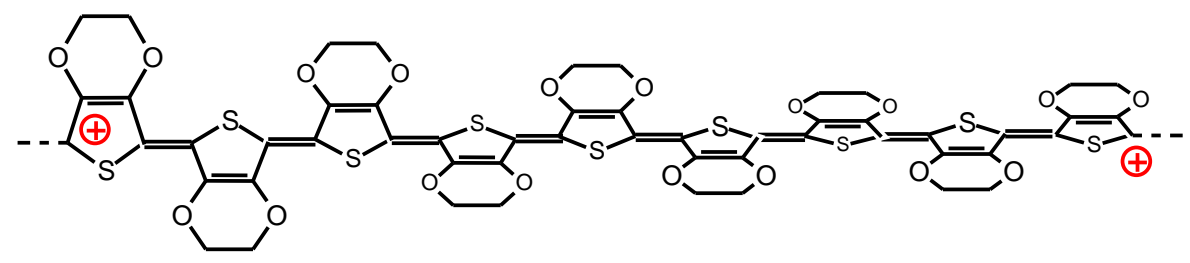

D)

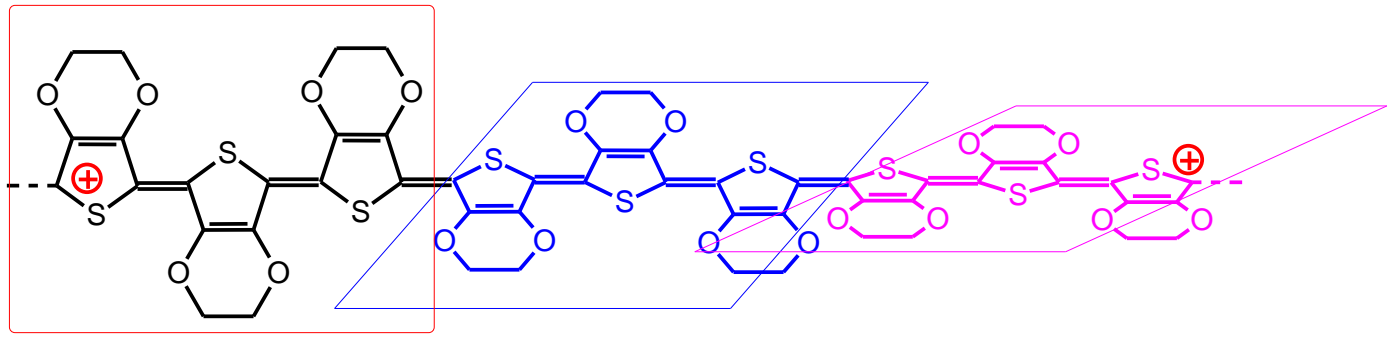

Figure 6. Possible structure of bipolarons in chiroptically active PEDOT* prepared in cholesteric liquid crystal. A) Planar structure; B) EDOT units gently twist in one-handed direction; C) neighboring 3,4-ethylene dioxythiophene (EDOT) units point oppositely and gently twist to form helical structure; D) three-EDOT units as a monomer repeat unit (terEDOT) gently twist in one direction to form helical structure.

The helical matrix of the cholesteric liquid crystal provides an asymmetric environment for the polymerization to produce helical polymers. The structural chirality is locked in by the insolubility and infusibility of the PEDOT* in the film state. If the

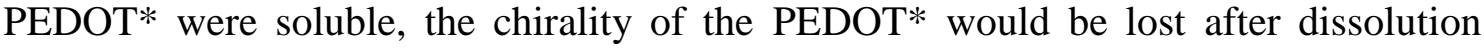
because the PEDOT* lacks chiral centers and unwinding of the helical structure results 


\section{Bipolaron Circular Dichroism in PEDOT*}

in racemization. Elasticity of the insoluble PEDOT* allows intrusion and extrusion of the dopant (intercalation) between the main-chains with maintenance of the structural chirality.

Chiral polythiophenes can form chiral aggregate structures. ${ }^{[17,18]}$ Formation of a helical aggregate structure is plausible, Fig 7(a). In this model, only low molecular weight part is referred for simple explanation. The electronic state of the PEDOT* in the neutral form was changed by doping because of the electron acceptor functionality of the dopant: doping produces polarons and bipolarons along the $\pi$-conjugated main-chain. ${ }^{[19]}$ Previous electron spin resonance (ESR) and optical rotation measurements of a polythiophene demonstrated that the generation of the charged species in the main-chain corresponded to a change in chiroptical activity and magnetic properties (electro-magnetic-chiroptical effect). ${ }^{[7]}$

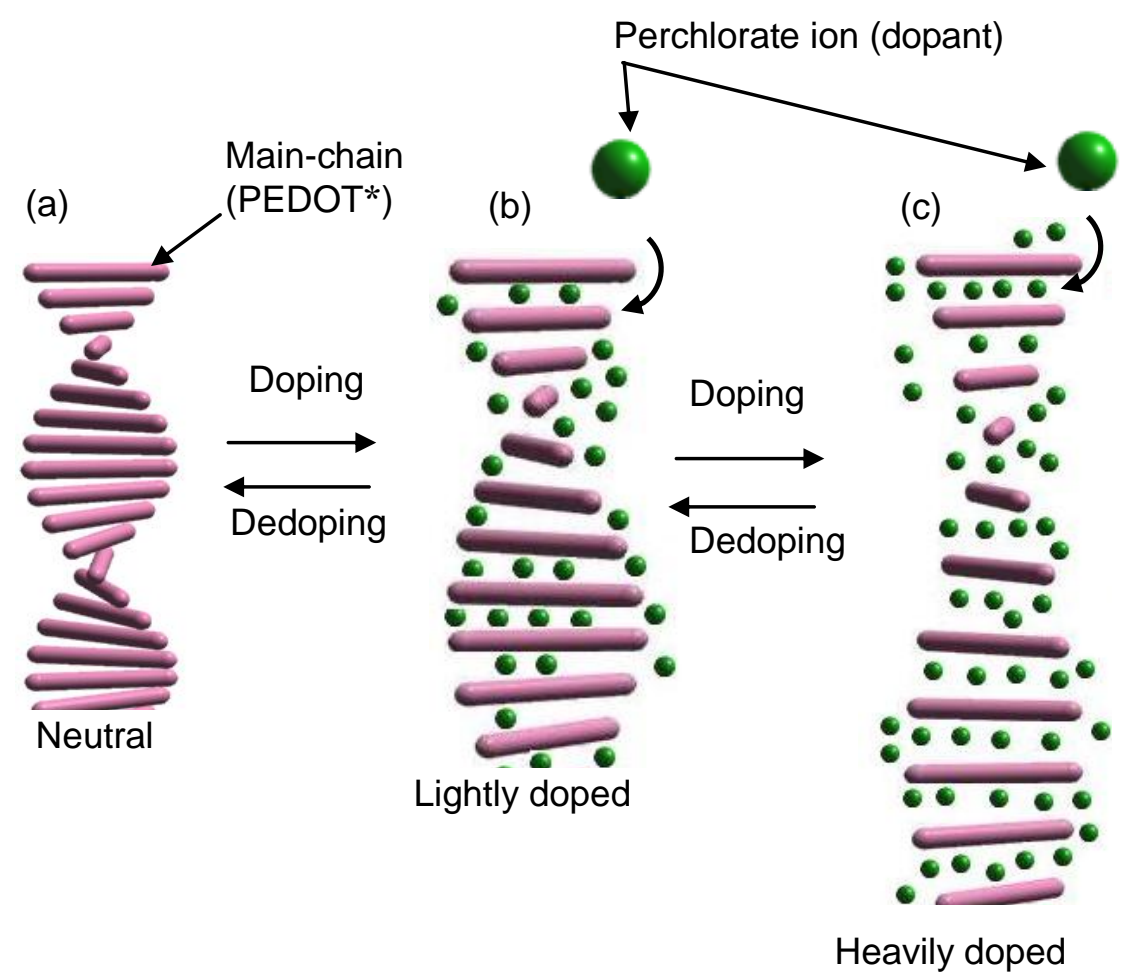

Figure 7. Proposed structures of doping for PEDOT* synthesized in the cholesteric liquid crystal. (a) Neutral state; (b) lightly doped; (c) heavily doped. The rods in (a)-(c) corresponds to the main-chain. In this case, the rods are low molecular weight fractions to express simple form of the aggregation. In practice, the rod length is very long in the case of high molecular weight fractions.

Doping expands the distance between the main-chains by intercalation of the perchlorate ions, Figs. 7(b,c), but the main-chains may still form helical aggregates. The contribution of the chiral aggregation factor to the entire chiroptical activity of the doped sample should be low. The chirality of the bipolaron state predominantly comes from the main-chain helical structure. The polarons and bipolarons consist of charged quinonoid structures. Certain "sequential units" of the quinonoid structure move along the main-chains as polarons and bipolarons. Here, polarons and bipolarons coexisted in the main-chains. The ratio of polarons/bipolarons is tuned by the doping level. The 


\section{Bipolaron Circular Dichroism in PEDOT*}

charged quinonoid unit are suggested to have gentle twists in a one-handed helical manner along the helically twisted main-chain, Fig. 8. We refer to chiroptical active polarons and bipolarons as "chiralions".

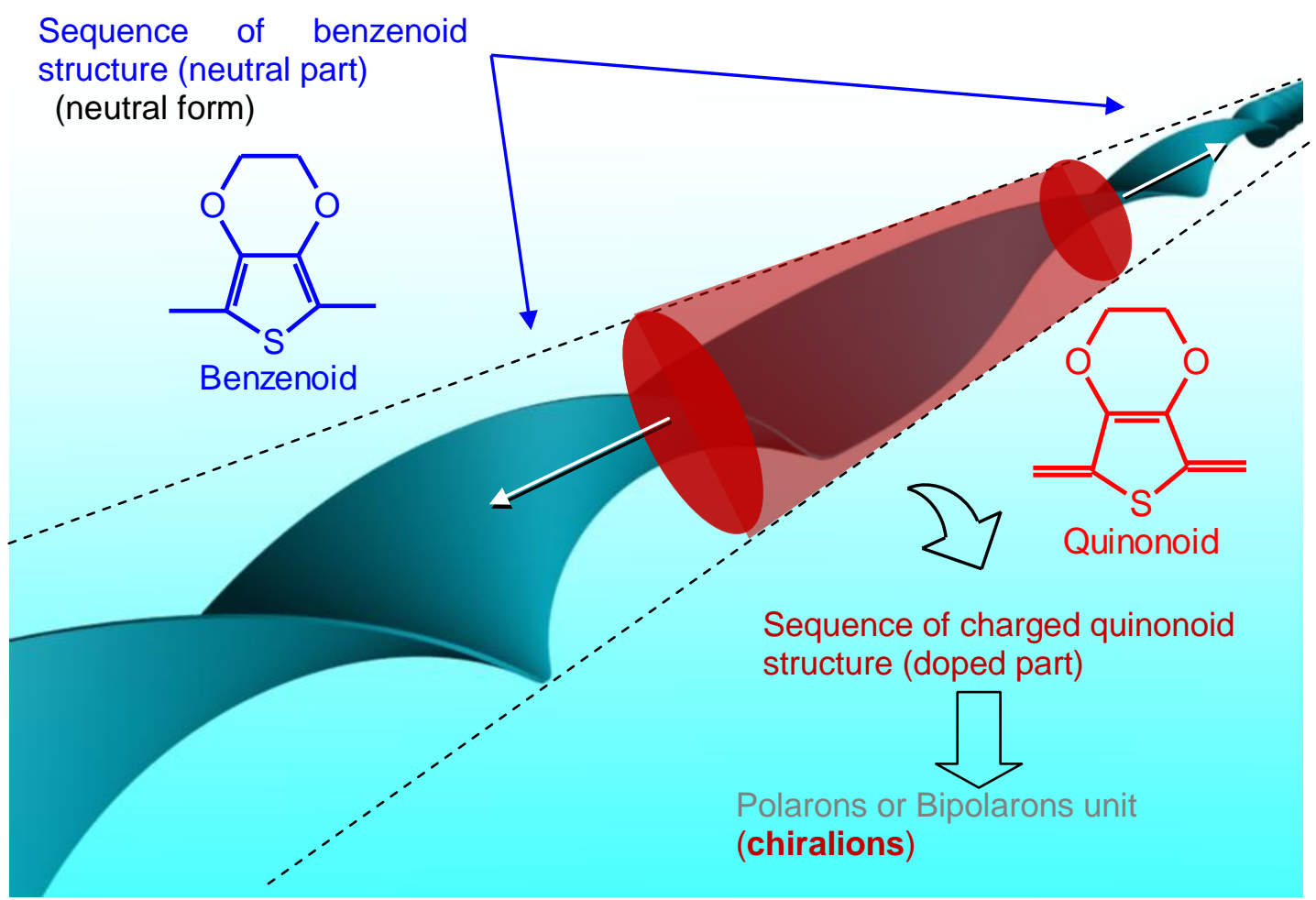

Figure 8. An illustration of sequences of benzenoid structure (neutral form) and quinonoid structure (polarons or bipolarons) with helicity.

The fingerprint macroscopic texture of the PEDOT*, produced by the matrix imprinting process, is not a necessary condition for the optical activities. We have previously reported the electro-chemically driven dynamic change in chiroptical activity of polyanilines bearing optically active camphor sulfonic acid groups via ionic bonds. ${ }^{[20]}$ Those polymers showed chiroptical activity without fingerprint textures. That result supports the view that the macroscopic fingerprint texture observable with SEM of the present PEDOT* was not a necessary condition to show chiroptical activities.

\section{Conclusions}

This research demonstrates that the PEDOT can form an atropisomer through formation of helical structures produced in cholesteric liquid crystals. A chiral-bipolaron state for the PEDOT* was confirmed through measurements of the NIR-CD, and chiral-bipolaron models were proposed. The chiroptical activity of the PEDOT* is suggested to be derived from its main-chain helicity. A modulation of the main-chain helicity, due to the generation of polarons and bipolarons, results in the electromagnetic-chiroptical effect of the PEDOT*. The macroscopic fingerprint texture observable under SEM is not a necessary condition to show chiroptical activities. 


\section{Bipolaron Circular Dichroism in PEDOT*}

However, one-handed main-chain helical structures at the molecular level are required to show chiroptical activities.

\section{Acknowledgments}

This research was supported by the Japan Society for the Promotion of Science (JSPS) Grant-in-Aid for Scientific Research \#25410218 (H. Goto). The author is grateful to JASCO Corp. (Japan) for the CD measurements, and Dr. M. Takeguchi (Microstructure Characterization Platform Promotion Office, National Institute for Materials Science, NIMS) for the SEM instrumentation and Dr. K. Kawabata (U. Tsukuba) (present: Yale U.) for helpful support in obtaining the SEM image (Fig 5(c)).

\section{References}

1. Sotzing, G. A.; Reddinger, J. L.; Katritzky, A. R.; Steel, P. J.; Soloducho, J.; Musgrave, R.;. Reynolds, J. R. Multiply colored electrochromic carbazole based polymers. Chem. Mater. 1997 9, $1578-1587$.

2. Sonmez, G.; Sonmez, H. B.; Shen, C. K. F.; Wudl, F. Red, green, and blue colors in polymeric electrochromics. Adv. Mater. 2004, 21, 1905-1908.

3. Beaujuge, P. M.; Ellinger S.; Reynolds, J. R. The donor-acceptor approach allows a black-to-transmissive switching polymeric electrochrome. Nature Mater. 2008, 7, 795-799.

4. Goto, H.; Kawabata, K. Light driven asymmetric polymerization: an approach for tele-control reaction. Polym. Chem. 2011, 2, 1098-1106.

5. Goto, H. Electrochemical polymerization in crystal - preparation of polybithiophene with crystal order. J. Polym. Sci. Part A: Polym. Chem. 2012, $50,622-628$.

6. Goto, H. An Optically Active Polythiophene Exhibiting Electrochemically Driven Light-Interference Modulation. Adv. Funct. Mater. 2009, 19, 1335-1342.

7. Goto, H. Optical Rotation and Electron Spin Resonance of an Electro-Optically Active Polythiophene. Mater. Chem. Phys. 2010, 122, 69-72.

8. Zhu, Y.; Heim I.; Tieke, B. Red emitting diphenylpyrrolopyrrole (DPP)-based polymers prepared by Stille and Heck coupling. Macromol. Chem. Phys., 2006, 207, 2206-2214.

9. Sotzing G. A.; Reynolds, J. R. Electrochromic conducting polymers via electrochemical polymerization of bis(2-(3,4-ethylenedioxy)thienyl) monomers Chem. Mater., 1996, 8, 882-889.

10. Raimundo, J.-M.; Blanchard, P.; Brisset, H.; Akoudad, S.; Roncali, J. Proquinoid acceptors as building blocks for the design of efficient $\pi$-conjugated fluorophores with high electron affinity. Chem. Commun., 2000, 939-940.

11. Goto, H. Vortex fibril structure and chiroptical electrochromic effect of optically active poly(3,4-ethylenedioxythiophene) (PEDOT*) prepared by chiral transcription electrochemical polymerisation in cholesteric liquid crystal. $J$. Mater. Chem. 2009, 19, 4914-4921.

12. Meng, H.; Perepichka, D. F.; Wudl, F. Facile solid-state synthesis of highly conducting poly(ethylenedioxythiophene), Angew. Chem., Int. Ed, 2003, 42, 658-661.

13. Roncali, J.; Blanchard, P.; Frère, P. 3,4-Ethylenedioxythiophene (EDOT) as a versatile building block for advanced functional p-conjugated systems. J. Mater. 


\section{Bipolaron Circular Dichroism in PEDOT*}

Chem., 2005, 15, 1589-1610.

14. Irvin, J. A.; Schwendeman, I.; Lee, Y.; Abboud, K. L.; Reynolds, J. R. Low-oxidation-potential conducting polymers derived from 3,4-ethylenedioxythiophene and dialkoxybenzenes. J. Polym. Sci. Part A: Polym. Chem. 2001, 39, 2164-2178.

15. Jackson, N. E.; Savoie, B. M.; Kohlstedt, K. L.; de la Cruz, M. O.; Schatz, G. C.; Chen, L. X.; Ratner, M. A. Controlling conformations of conjugated polymers and small molecules: The role of nonbonding interactions. J. Am. Chem. Soc. 2013, 135, 10475-10483.

16. C. X. Cui, M. Kertesz. Two helical conformations of polythiophene, polypyrrole, and their derivatives. Phys. Rev. B, 1989, 40, 9661-9670.

17. Langeveld-Voss, B.M.W.; Janssen, R.A.J.; Meijer, E.W. On the origin of optical activity in polythiophenes. J. Mol. Struct. 2000, 521, 285-301.

18. Matthews, J. R. F.; Goldoni, H.; Kooijman, Spek, A. L.; Schenning, A. P. H. J.; Meijer, E. W. Metal coordination and aggregation properties of chiral polythiophenes and polythienylethynylenes. Macromol. Rapid Commun. 2007, 28, 1809-1815.

19. Furukawa, Y. Electronic Absorption and vibrational spectroscopies of conjugated conducting polymers. Y. J. Phys. Chem. 1996, 100, 15644.

20. Goto, H. Optically active electrochromism in polyanilines. J. Polym. Sci. Part A. Polym. Chem. 2007, 45, 2085-2090. 